\title{
Action to Support Practices Implement Research Evidence (ASPIRE): protocol for a cluster-randomised evaluation of adaptable implementation packages targeting 'high impact' clinical practice recommendations in general practice
}

Thomas A. Willis ${ }^{1 *}$ D , Suzanne Hartley², Liz Glidewell1', Amanda J. Farrin², Rebecca Lawton ${ }^{3,4}$, Rosemary R. C. McEachan ${ }^{4}$, Emma Ingleson', Peter Heudtlass², Michelle Collinson², Susan Clamp', Cheryl Hunter ${ }^{1}$, Vicky Ward ${ }^{1}$, Claire Hulme ${ }^{1}$, David Meads ${ }^{1}$, Daniele Bregantini ${ }^{1}$, Paul Carder $^{5}$, Robbie Foy ${ }^{1}$ and on behalf of the ASPIRE programme

\footnotetext{
Abstract

Background: There are recognised gaps between evidence and practice in general practice, a setting which provides particular challenges for implementation. We earlier screened clinical guideline recommendations to derive a set of 'high impact' indicators based upon criteria including potential for significant patient benefit, scope for improved practice and amenability to measurement using routinely collected data. We aim to evaluate the effectiveness and costeffectiveness of a multifaceted, adaptable intervention package to implement four targeted, high impact recommendations in general practice.

(Continued on next page)
}

\footnotetext{
* Correspondence: t.a.willis@leeds.ac.uk

${ }^{1}$ Leeds Institute of Health Sciences, University of Leeds, Leeds LS2 9JT, UK

Full list of author information is available at the end of the article
} 
(Continued from previous page)

Methods/design: The research programme Action to Support Practice Implement Research Evidence (ASPIRE) includes a pair of pragmatic cluster-randomised trials which use a balanced incomplete block design. Clusters are general practices in West Yorkshire, United Kingdom (UK), recruited using an 'opt-out' recruitment process. The intervention package adapted to each recommendation includes combinations of audit and feedback, educational outreach visits and computerised prompts with embedded behaviour change techniques selected on the basis of identified needs and barriers to change. In trial 1, practices are randomised to adapted interventions targeting either diabetes control or risky prescribing and those in trial 2 to adapted interventions targeting either blood pressure control in patients at risk of cardiovascular events or anticoagulation in atrial fibrillation. The respective primary endpoints comprise achievement of all recommended target levels of haemoglobin A1c ( $\mathrm{HbA1c}$ ), blood pressure and cholesterol in patients with type 2 diabetes, a composite indicator of risky prescribing, achievement of recommended blood pressure targets for specific patient groups and anticoagulation prescribing in patients with atrial fibrillation. We are also randomising practices to a fifth, non-intervention control group to further assess Hawthorne effects. Outcomes will be assessed using routinely collected data extracted 1 year after randomisation. Economic modelling will estimate intervention cost-effectiveness. A process evaluation involving eight non-trial practices will examine intervention delivery, mechanisms of action and unintended consequences.

Discussion: ASPIRE will provide 'real-world' evidence about the effects, cost-effectiveness and delivery of adapted intervention packages targeting high impact recommendations. By implementing our adaptable intervention package across four distinct clinical topics, and using 'opt-out' recruitment, our findings will provide evidence of wider generalisability.

Trial registration: ISRCTN91989345

Keywords: Primary care, Implementation, Cluster-randomised trial, Clinical guidelines, Diabetes, Prescribing, Atrial fibrillation

\section{Background}

Clinical research can only benefit patient and population health if findings are incorporated into routine care. There are widely recognised failures to introduce effective new clinical practices quickly enough, consistently use those already proven to be effective or stop those found to be ineffective or even harmful. The gap between evidence and practice is an important problem for policy-makers, healthcare systems and research funders because it limits the health, social and economic impacts of clinical research [1]. Dissemination of best practice is necessary but seldom sufficient by itself to ensure implementation.

The context of general practice in the United Kingdom (UK) presents particular implementation challenges-given the limited practice organisational capacity, the increasing complexity of care and the dispersed and independent nature of practices. Many implementation studies focus on one condition (e.g. depression, back pain). This limits generalisability; it is uncertain how an intervention developed for one clinical condition will work for another [2, 3]. In 2012, we identified 107 clinical guidelines relevant to general practice produced by the National Institute for Health and Care Excellence (NICE) [4]. It is impracticable and inefficient to invent an implementation strategy for every new guideline. Implementation strategies are required which can be adapted to a range of targeted problems and sustainably integrated into available primary care systems and resources [5].

Action to Support Practices Implement Research Evidence (ASPIRE) is a research programme that aims to develop and evaluate an adaptable intervention package to target implementation of 'high impact' clinical practice recommendations in general practice. ASPIRE comprises five, sequential work packages:

1. Screening of NICE guidelines and associated quality standards to derive a set of 'high impact' indicators based on burden of illness, potential for significant patient benefit from improved practice, likelihood of cost savings without patient harm and feasibility of measuring change using routinely collected data [4].

2. Cross-sectional analysis of patient data to identify high impact recommendations with greatest scope for improvement (low adherence) and explore variations in adherence.

3. Interviews with primary care professionals to explore barriers to and enablers of adherence to selected high impact recommendations, matching of behaviour change techniques to identified barriers and enablers and development of an adaptable intervention package (based on audit and feedback, outreach educational visits and computerised prompts). 
4. Evaluation of the effectiveness and cost-effectiveness of the adapted intervention package in targeting the implementation of high impact recommendations.

5. Conduct of a parallel process evaluation to examine intervention delivery, mechanisms of action and unintended consequences.

This protocol paper describes the randomised evaluation and summarises the process evaluation.

\section{Methods/design}

\section{Study design and setting}

We are conducting a pair of pragmatic clusterrandomised trials with general practices as the unit of allocation. Cluster randomisation was chosen because the intervention is delivered at the practice level and aims to change clinical practice of the whole practice team. Balanced incomplete block designs equalise Hawthorne effects whilst maximising power and efficiency $[6,7]$ and also reduce the risk of overburdening practices through exposure to more than one intervention. In trial 1, practices will be randomised to adapted intervention packages targeting either diabetes control or risky prescribing. In trial 2, practices will be randomised to adapted intervention packages targeting either blood pressure control in patients at high risk of cardiovascular events or anticoagulation in atrial fibrillation. The balanced incomplete block design has two treatment arms per trial. Within both trials, all intervention packages were assumed to be independent in terms of their outcomes and each treatment arm will therefore be used as the control arm for the other treatment arm in the same trial (Fig. 1 and Additional file 1 detail a full CONSORT checklist). We are also including a fifth, non-intervention control group to further assess Hawthorne effects. Practices allocated to this arm will not receive any of the ASPIRE interventions, but we will examine outcomes via the same routinely collected data.

The study is set within West Yorkshire, which covers a socioeconomically and ethnically diverse population of approximately 2.2 million residents [8]. Around 330 general practices are organised within 10 clinical commissioning groups (CCGs), which have roles including the purchasing of specialist services and improving quality of primary care.

\section{Participants}

General practices are eligible if they used the SystmOne computerised clinical system (TPP, http://www.tpp-

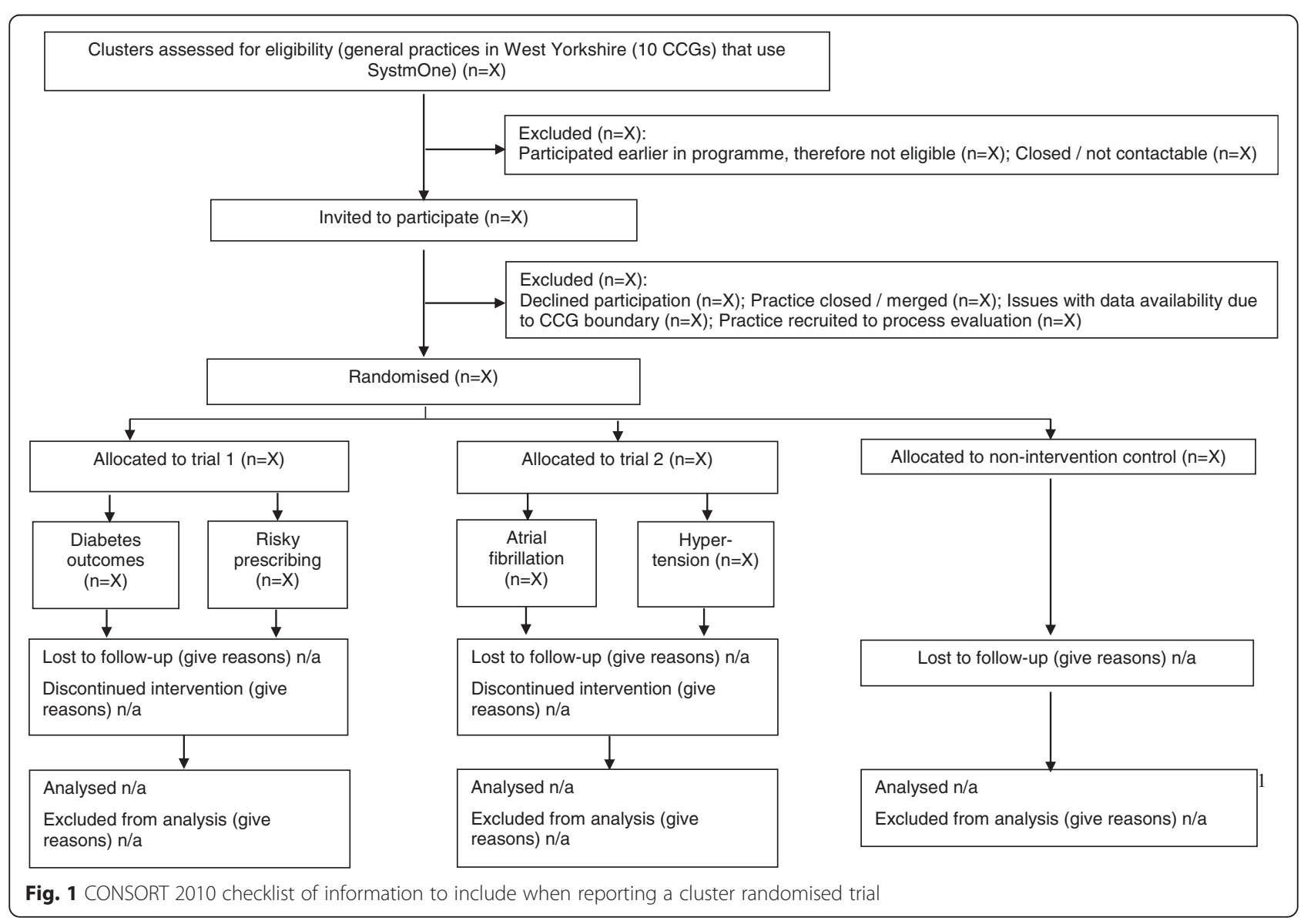


uk.com/). Approximately two thirds of West Yorkshire practices use SystmOne. The use of a single system simplifies the process of data extraction and of implementing software support. We will exclude practices which had been involved in earlier stages of intervention development and piloting. We will also exclude from trial 2 practices in one CCG involved in a concurrent initiative addressing anticoagulation in atrial fibrillation.

\section{Recruitment and consent}

Following consultations with our Programme Steering Group, our Public and Patient Involvement Panel and all 10 CCGs, we will use an 'opt-out' approach to recruit and consent general practices. Apart from facilitating the recruitment process, we judge that opt-out recruitment will enhance pragmatism and hence generalisability by resembling recruitment processes typically used by routine quality improvement initiatives [9]. We will send all eligible practices an invitation and information sheet via email. This outlines the purpose of the trial and what involvement entails and states that practices will be included unless they indicate otherwise. We will follow this with a duplicate pack (including a Freepost return envelope to return the opt-out notification) sent via recorded postal delivery. After 2 weeks, we will send reminders by both email and recorded postal delivery to all nonresponding practices. Four weeks post-initial invitation, we will consider all practices which have not opted-out to have consented to participate. Patient-level consent is not required as all patient-level data are anonymised.

\section{Intervention}

We broadly conceptualise the implementation intervention as comprising a range of behaviour change techniques (e.g. social comparison, information on health consequences, action planning). These will be embedded within a package based around three delivery mechanisms that possess a known evidence base and are increasingly central to routine implementation activities [7]: audit and feedback [7, 10], educational outreach visits $[7,11]$ and computerised prompts [7, 12]. We have further developed the packages iteratively through a series of exchanges with our professional and patient advisory groups and piloting in five general practices.

Following earlier work and consultations with professional and patient advisory groups, we decided to target the implementation of four high impact indicators:

- Control of HbA1c, blood pressure and cholesterol in type 2 diabetes $[13,14]$

- Risky prescribing, largely focusing on avoiding adverse effects of non-steroidal anti-inflammatory drugs (NSAIDs) and anti-platelet drugs [15]
- Control of blood pressure in people at high risk of cardiovascular events [16] and

- Anticoagulation in people with atrial fibrillation [17].

We conducted semi-structured interviews with primary care professionals based on the Theoretical Domains Framework [18] to explore barriers and enablers to achieving the above indicators. For each indicator, we matched behaviour change techniques targeting clinicians to the most salient barriers and enablers and embedded them within intervention packages.

In brief, the audit and feedback component will comprise quarterly reports individualised for each practice and presenting their performance against targeted clinical indicators, together with motivational messages based on key theoretical domains identified by our qualitative research. The educational outreach visits will be delivered by trained pharmacist facilitators and involve discussion of practice performance and development of action plans. The computerised support will include modified versions of the searches used to prepare the audit reports and, for risky prescribing only, automated messages triggered by potentially risky prescribing combinations. We will also offer practices up to 2 days of additional pharmacist support. The nature of this support will be decided in discussion with practice but can include review of patient notes or modification of computerised searches to identify specific patient groups to invite for consultation. All practices in the intervention arms will be sent electronic and postal copies of the feedback reports. They will also be offered educational outreach, additional pharmacist support, searches to identify patients and, where applicable, computerised prompts. Given the pragmatic nature of these trials, the acceptance and engagement with all components is optional. We will further describe intervention development and content in a separate paper.

\section{Outcomes}

The primary endpoints are as follows:

- The proportion of patients with type 2 diabetes achieving all three of the following treatment targets: blood pressure below 140/80 $\mathrm{mmHg}$ (or $130 / 80 \mathrm{mmHg}$ if there is kidney, eye or cerebrovascular damage), HbA1c value below or equal to $59 \mathrm{mmol} / \mathrm{mol}$ and cholesterol level below or equal to $5.0 \mathrm{mmol} / \mathrm{l}$ (trial 1 )

- A composite measure of nine indicators of high-risk NSAID and anti-platelet prescribing: prescribing a traditional oral NSAID or low-dose aspirin in patients with a history of peptic ulceration without co-prescription of gastro-protection; prescribing a traditional oral NSAID in patients aged 75 years or 
over without co-prescription of gastro-protection; prescribing of a traditional oral NSAID and aspirin in patients aged 65 years or over without co-prescription of gastro-protection; prescribing of aspirin and clopidogrel in patients aged 65 years or over without co-prescription of gastro-protection; prescribing of warfarin and a traditional oral NSAID; prescribing of warfarin and low-dose aspirin or clopidogrel without co-prescription of gastro-protection; prescribing an oral NSAID in patients with heart failure; prescribing an oral NSAID in patients prescribed both a diuretic and an angiotensin-converting-enzyme inhibitor (ACE-I) or angiotensin receptor blocker (ARB) and prescribing an oral NSAID in patients with chronic kidney disease (CKD; trial 1)

- The proportion of patients with satisfactorily controlled blood pressure according to recommended targets: under 140/90 $\mathrm{mmHg}$ in patients aged under 80 years with hypertension; under 150/90 $\mathrm{mmHg}$ in patients aged 80 years and over with hypertension; under $140 / 80 \mathrm{mmHg}$ in patients aged under 80 years with diabetes, under $130 / 80 \mathrm{mmHg}$ if there are complications of diabetes; under 130/80 $\mathrm{mmHg}$ in patients aged under 80 years with chronic kidney disease and proteinuria; under 140/90 $\mathrm{mmHg}$ in patients aged under 80 years with coronary heart disease; under $140 / 90 \mathrm{mmHg}$ in patients aged under 80 years with peripheral arterial disease; under 140/90 $\mathrm{mmHg}$ in patients aged under 80 years with a history of stroke/ transient ischemic attack and under $140 / 90 \mathrm{mmHg}$ in patients aged under 80 years with a cardiovascular disease risk of $20 \%$ or higher (trial 2) and

- A composite of the proportion of men with atrial fibrillation and a $\mathrm{CHA}_{2} \mathrm{DS}_{2}$-VASc score of 1 prescribed anticoagulation therapy and the proportion of all people with atrial fibrillation and a $\mathrm{CHA}_{2} \mathrm{DS}_{2}$-VASc score of 2 or above prescribed anticoagulation therapy (trial 2).

Secondary endpoints comprise the following:

- The effects on separate individual indicators that contribute to composite outcomes (e.g. individual blood pressure, HbA1c and cholesterol targets in patients with diabetes)

- The effects on recorded processes of care (the proportion of patients with type 2 diabetes achieving all nine of the following recommended processes of care in the previous 12 months: blood pressure recording, HbA1c recording, total cholesterol recording, urine albumin to creatinine ratio (ACR) or protein to creatinine ratio (PCR) or proteinuria coding, estimated glomerular filtration rate (eGFR) or serum creatinine testing, foot care review, retinal screening, body mass index recording, smoking status)

- The effects on continuous intermediate clinical outcomes (i.e. value of last recorded blood pressure, HbA1c, cholesterol)

In addition, we will gather selected data from the Quality and Outcomes Framework (QOF; 2015-2016 indicator list (Table 1). The QOF is a performance management system whereby general practices are remunerated according to achievement of targets reflecting quality of care across four domains of clinical, organisational, patient experience and additional services [19]. Practice data collection for QOF operates on an annual cyclical basis from 1 April to 31 March. Several indicators map onto our trial recommendations (Table 1). We have also selected a series of non-trial-related indicators that represent different aspects of care and service delivery. These will be used to measure unintended impacts on quality of care and relate to four areas: coronary heart disease (an example of a long-term physical condition), mental health (long-term non-physical condition), smoking (health promotion) and asthma (long-term physical condition). All outcomes will be assessed at 1 year following randomisation.

\section{Data collection}

\section{Primary and secondary endpoints}

We will use anonymised patient health records, extracted remotely from participating practices to obtain data on indicator adherence. The data will be collected via the data quality team at the National Health Service (NHS) Yorkshire \& Humber Commissioning Support Unit. Data extraction and collation procedures are performed under the appropriate Information Governance Guidance, in line with Caldicott Principles.

Data queries are developed by mapping inclusion and exclusion criteria to the numerators and denominators for each indicator and building the search within SystmOne. Reports are generated by a data analyst to define output for each indicator numerator and denominator and extracted in a standardised format. The outputs are anonymised and checked for accuracy prior to transfer to the Leeds Institute of Clinical Trials Research (LICTR) via a secure file transfer system.

Pre-intervention data will be extracted for randomisation stratification and then continue quarterly to inform the content of feedback reports. The final extraction will be used for endpoint analysis. 
Table 1 List of indicators from the Quality and Outcomes Framework 2015-2016 included in the ASPIRE analysis

\begin{tabular}{|c|c|c|}
\hline Domain & $\begin{array}{l}\text { QOF indicator number } \\
\text { (2015-2016) }\end{array}$ & Indicator \\
\hline Atrial fibrillation & AF006 & $\begin{array}{l}\text { The percentage of patients with atrial fibrillation in whom stroke risk has been assessed } \\
\text { using the CHA2DS2-VASc score risk stratification scoring system in the preceding } 12 \text { months } \\
\text { (excluding those patients with a previous CHADS2 or CHA2DS2-VASc score of } 2 \text { or more). }\end{array}$ \\
\hline Atrial fibrillation & AF007 & $\begin{array}{l}\text { In those patients with atrial fibrillation with a record of a CHA2DS2-VASC score of } 2 \text { or more, } \\
\text { the percentage of patients who are currently treated with anticoagulation drug therapy. }\end{array}$ \\
\hline $\begin{array}{l}\text { Secondary prevention of } \\
\text { coronary heart disease }\end{array}$ & CHD002 & $\begin{array}{l}\text { The percentage of patients with coronary heart disease in whom the last blood pressure } \\
\text { reading (measured in the preceding } 12 \text { months) is } 150 / 90 \mathrm{mmHg} \text { or less. }\end{array}$ \\
\hline $\begin{array}{l}\text { Secondary prevention of } \\
\text { coronary heart disease }\end{array}$ & CHD005 & $\begin{array}{l}\text { The percentage of patients with coronary heart disease with a record in the preceding } \\
12 \text { months that aspirin, an alternative anti-platelet therapy or an anti-coagulant is being taken. }\end{array}$ \\
\hline $\begin{array}{l}\text { Secondary prevention of } \\
\text { coronary heart disease }\end{array}$ & CHD007 & $\begin{array}{l}\text { The percentage of patients with coronary heart disease who have had influenza immunisation } \\
\text { in the preceding } 1 \text { August to } 31 \text { March. }\end{array}$ \\
\hline Hypertension & HYP006 & $\begin{array}{l}\text { The percentage of patients with hypertension in whom the last blood pressure reading } \\
\text { (measured in the preceding } 12 \text { months) is } 150 / 90 \mathrm{mmHg} \text { or less. }\end{array}$ \\
\hline $\begin{array}{l}\text { Stroke and transient } \\
\text { ischemic attack }\end{array}$ & STIA003 & $\begin{array}{l}\text { The percentage of patients with a history of stroke or TIA in whom the last blood } \\
\text { pressure reading (measured in the preceding } 12 \text { months) is } 150 / 90 \mathrm{mmHg} \text { or less. }\end{array}$ \\
\hline Diabetes mellitus & DM002 & $\begin{array}{l}\text { The percentage of patients with diabetes, on the register, in whom the last } \\
\text { blood pressure reading (measured in the preceding } 12 \text { months) is } 150 / 90 \mathrm{mmHg} \text { or less. }\end{array}$ \\
\hline Diabetes mellitus & DM003 & $\begin{array}{l}\text { The percentage of patients with diabetes, on the register, in whom the last blood pressure } \\
\text { reading (measured in the preceding } 12 \text { months) is } 140 / 80 \mathrm{mmHg} \text { or less. }\end{array}$ \\
\hline Diabetes mellitus & DM004 & $\begin{array}{l}\text { The percentage of patients with diabetes, on the register, whose last measured } \\
\text { total cholesterol (measured within the preceding } 12 \text { months) is } 5 \mathrm{mmol} / \mathrm{l} \text { or less. }\end{array}$ \\
\hline Diabetes mellitus & DM006 & $\begin{array}{l}\text { The percentage of patients with diabetes, on the register, with a diagnosis of } \\
\text { nephropathy (clinical proteinuria) or micro-albuminuria who are currently } \\
\text { treated with ACE-I (or ARBs). }\end{array}$ \\
\hline Diabetes mellitus & DM007 & $\begin{array}{l}\text { The percentage of patients with diabetes, on the register, in whom the last IFCC-HbA1c is } \\
59 \mathrm{mmol} / \mathrm{mol} \text { or less in the preceding } 12 \text { months. }\end{array}$ \\
\hline Diabetes mellitus & DM008 & $\begin{array}{l}\text { The percentage of patients with diabetes, on the register, in whom the last IFCC-HbA1c is } \\
64 \mathrm{mmol} / \mathrm{mol} \text { or less in the preceding } 12 \text { months. }\end{array}$ \\
\hline Diabetes mellitus & DM009 & $\begin{array}{l}\text { The percentage of patients with diabetes, on the register, in whom the last IFCC-HbA1c is } \\
75 \mathrm{mmol} / \mathrm{mol} \text { or less in the preceding } 12 \text { months. }\end{array}$ \\
\hline Diabetes mellitus & DM0012 & $\begin{array}{l}\text { The percentage of patients with diabetes, on the register, with a record of a foot } \\
\text { examination and risk classification: (1) low risk (normal sensation, palpable pulses), } \\
\text { (2) increased risk (neuropathy or absent pulses), (3) high risk (neuropathy or absent } \\
\text { pulses plus deformity or skin changes in previous ulcer) or (4) ulcerated foot within } \\
\text { the preceding } 12 \text { months. }\end{array}$ \\
\hline Diabetes mellitus & DM0014 & $\begin{array}{l}\text { The percentage of patients newly diagnosed with diabetes, on the register, in preceding } \\
1 \text { April to } 31 \text { March who have a record of being referred to a structured education } \\
\text { programme within } 9 \text { months after entry on to the diabetes register }\end{array}$ \\
\hline Diabetes mellitus & DM0018 & $\begin{array}{l}\text { The percentage of patients with diabetes, on the register, who have had influenza } \\
\text { immunisation in the preceding } 1 \text { August to } 31 \text { March. }\end{array}$ \\
\hline Mental health & MH002 & $\begin{array}{l}\text { The percentage of patients with schizophrenia, bipolar affective disorder and other psychoses } \\
\text { who have a comprehensive care plan documented in the record, in the preceding } 12 \text { months, } \\
\text { agreed between individuals, their family and/or carers as appropriate. }\end{array}$ \\
\hline Mental health & MH003 & $\begin{array}{l}\text { The percentage of patients with schizophrenia, bipolar affective disorder and other } \\
\text { psychoses who have a record of blood pressure in the preceding } 12 \text { months. }\end{array}$ \\
\hline Smoking & SMOK002 & $\begin{array}{l}\text { The percentage of patients with any or any combination of the following conditions: coronary } \\
\text { heart disease (CHD), peripheral arterial disease (PAD), stroke or transient ischemic attack (TIA), } \\
\text { hypertension, diabetes, chronic obstructive pulmonary disease (COPD), chronic kidney disease } \\
\text { (CKD), asthma, schizophrenia, bipolar affective disorder or other psychoses whose notes record } \\
\text { smoking status in the preceding } 12 \text { months. }\end{array}$ \\
\hline
\end{tabular}


Table 1 List of indicators from the Quality and Outcomes Framework 2015-2016 included in the ASPIRE analysis (Continued)

\begin{tabular}{lll}
\hline Smoking & SMOKO04 & $\begin{array}{l}\text { The percentage of patients aged } 15 \text { or over who are recorded as current smokers who have a } \\
\text { record of an offer or support and treatment within the preceding } 24 \text { months. } \\
\text { Smoking } \\
\text { SMOK005 }\end{array}$ \\
& $\begin{array}{l}\text { The percentage of patients with any or any combination of the following conditions: CHD, PAD, } \\
\text { stroke or TIA, hypertension, diabetes, COPD, CKD, asthma, schizophrenia, bipolar } \\
\text { affective disorder or other psychoses who are recorded as current } \\
\text { smokers who have a record of an offer of support and treatment } \\
\text { within the preceding } 12 \text { months }\end{array}$ \\
AST003 & $\begin{array}{l}\text { The percentage of patients with asthma, on the register, who have had an asthma } \\
\text { review in the preceding } 12 \text { months that includes an assessment of asthma control } \\
\text { using the three Royal College of Physician questions. }\end{array}$ \\
\end{tabular}

\section{Practice and patient characteristics}

Data on practice characteristics will be obtained from publicly available sources (http://www.hscic.gov.uk) and include practice list size (number of registered patients), number of general practitioner (GP) partners, number of salaried GPs, practice teaching status, practice level Index of Multiple Deprivation (IMD), ethnic profile of practice register, achievement of QOF indicators, patient satisfaction (proportion who would recommend practice to others), patient-rated practice accessibility (proportion able to speak with GP or nurse within 48 hours of approach) and practice prescribing costs. Patient characteristics will be extracted alongside adherence data and include age, sex, co-morbidity (number of QOF disease registers patient is included in) and polypharmacy (number of repeat prescriptions).

\section{Intervention fidelity}

Intervention delivery and fidelity will be monitored throughout the trials. Structured logs record the number of outreach sessions delivered, number of staff attending and whether feedback reports were (i) received and (ii) used, as well as any additional support requested by the practice. Outreach facilitators will complete standard review forms following each visit. Reasons for declining an outreach visit will also be documented. We will collect data on which practices accept the invitation to join indicator-specific SystmOne organisational groups. Acceptance is required in order to access the searches, as well as any available computer protocols. These lists will be monitored during the trials and reminders sent to practices which have not joined.

\section{Sample size}

Data collected for the second ASPIRE work package (cross-sectional assessment in a random sample of practices of adherence to selected clinical indicators) was used to estimate mean cluster size (number of targeted patients per practice by indicator), coefficient of variation, intra-cluster correlation coefficient and control group achievement rates (Table 2). Depending on the recommendation, assumed intra-cluster correlation coefficients (ICC) ranged from 0.03 to 0.06 , mean cluster sizes from 55 to 800 and the coefficient of variation of cluster sizes from 0.6 to 0.79 (Table 2). The median effect sizes on processes and outcomes of care for a range of single interventions in guideline implementation studies are around 4-9\% [20, 21]. Given the enhancements we are making to the intervention packages and that we are targeting recommendations with greater scope for improvement [10], we judge that an estimated effect size of $15 \%$ for outcomes related to diabetes, hypertension and atrial fibrillation are realistic and clinically relevant. Control group adherence rates in risky prescribing are considerably higher (Table 2), and considering a potential ceiling effect, we estimated $5 \%$ to be a realistic and clinically relevant effect size for this intervention.

In order to achieve $90 \%$ power, and allowing for an alpha error rate of $2.5 \%$ (to adjust for two outcome comparisons in each trial) and a $10 \%$ drop-out rate, we require 40 clusters per arm in trial 1 (diabetes and risky prescribing) and 32 clusters per arm in trial 2 (hypertension and atrial fibrillation). We therefore aim to recruit 144 practices (Table 3). We allowed for the possibility of a fifth arm because we anticipated achieving above-target recruitment levels using the opt-out approach.

\section{Randomisation}

Randomisation will be conducted using a computergenerated minimisation programme (incorporating a random element) to ensure arms are balanced. It will follow a two-stage process. First, practices will be stratified by CCG and practice list size (defined as above/ below the West Yorkshire median list size of 6562 patients) and then randomised to trial 1 , trial 2 or the no-intervention arm. Second, practices in trials 1 and 2 will then be stratified again by CCG, practice list size and pre-intervention adherence to the two relevant targeted clinical areas. They will then be randomised to the individual arms within each trial. Cluster randomisation will be performed at LICTR by the trial statistician.

For both trials, each practice will act as a control practice for the other corresponding arm in the trial to which they are randomised. Practices in the no-intervention arm will operate as additional controls. 
Table 2 Key sample size assumptions

\begin{tabular}{lllll}
\hline & Atrial fibrillation & Blood pressure control & Risky prescribing & Diabetes control \\
\hline Mean number of patients per practice (cluster size) & 55 & 800 & 420 & 280 \\
Coefficient of variation (CV) of cluster size & 0.79 & 0.67 & 0.65 & 0.6 \\
Intra-cluster correlation coefficient (ICC) & 0.06 & 0.06 & 0.03 & 0.06 \\
Control group adherence & 0.6 & 0.72 & 0.89 & 0.43 \\
\hline
\end{tabular}

General practice staff and trial personnel involved in the delivery of the intervention will, of necessity, be aware of assignment to allocation but collection of outcomes for the primary endpoints will be blind.

\section{Statistical analysis}

All analyses and data summaries will be conducted on the intention-to-treat (ITT) population, defined as all randomised practices regardless of compliance with the intervention or withdrawal from the study. No formal interim analyses are planned, and final analysis will take place when all available data are received from the final extract.

\section{Primary analysis}

As the trials are cluster randomised, the primary outcome measures (levels of adherence to the four indicators) will be compared between the intervention and control groups using two-level binary logistic models, with patients nested within general practices. Effect sizes and $95 \%$ confidence intervals will be reported.

\section{Secondary analyses}

Secondary analyses will be undertaken using both binary and continuous outcome data (e.g. blood pressure levels). For binary outcome data, we will also be using two-level binary logistic models, for continuous data, two-level linear models. Effect sizes and $95 \%$ confidence intervals will be reported.

\section{Cost-effectiveness analysis}

We plan to conduct economic analyses for three of the four intervention packages: diabetes control, risky prescribing and blood pressure control. We had to limit the number of outcomes modelled because of our limited resources and selected three based on availability of existing models.
Economic modelling has the primary objective of identifying the interventions' incremental cost-effectiveness ratios. In order to assess the impact of the intervention over a lifetime horizon, longer-term models will be developed for each of the three selected areas. The decision analytic models will be informed by published evidence on costs (taking a UK health and social care perspective as per NICE guidelines) and utility values (used to construct quality-adjusted life years [QALYs]). Values generated for time periods greater than 12 months will be discounted at the $3.5 \%$ rate.

The economic evaluations will make use of an adherence parameter informed by effectiveness data obtained from the study. The parameter will reflect the impact of the intervention; for example, an improvement in adherence should lead to an improvement in outcomes (QALYs) predicted by the model.

The value of the intervention will depend upon the value of the behaviour it promotes. Thus, its costeffectiveness will be application-specific, just as drugs which treat multiple conditions do not have a single costeffectiveness. The main outcome of interest will be cost per QALY and net monetary benefit. We will conduct extensive deterministic sensitivity analysis and probabilistic sensitivity analysis to assess uncertainty surrounding estimates of cost-effectiveness.

\section{Trial and data monitoring}

Trial supervision includes a core project team, a Trial Management Group (TMG; consisting of a subset of the project team and relevant independent experts in statistics and health economics) and an independent Trial Steering Committee (TSC). For a trial of this nature and duration, a separate Data Monitoring and Ethics Committee is not required. Rather, the TSC will adopt a safety monitoring role, with the constitution of a subcommittee to review safety issues where this becomes necessary. Any clinical governance issues pertaining to

Table 3 Trial design and number of practices required

\begin{tabular}{lllll}
\hline Trial 1 & & Trial 2 & Non-intervention control \\
\cline { 1 - 2 } $\begin{array}{llll}\text { Diabetes } \\
\text { control }\end{array}$ & $\begin{array}{l}\text { Risky } \\
\text { prescribing }\end{array}$ & $\begin{array}{l}\text { Blood pressure } \\
\text { control }\end{array}$ & $\begin{array}{l}\text { Anticoagulation in atrial } \\
\text { fibrillation }\end{array}$ & \\
\hline 40 & 40 & 32 & 32 & $\begin{array}{l}\text { [Number dependent on recruitment beyond that required for } \\
\text { intervention sample] }\end{array}$ \\
\hline
\end{tabular}


aspects of routine management will be brought to the attention of the TSC and, where applicable, to individual practices.

\section{Process evaluation}

We will conduct a process evaluation alongside the trials to:

- Describe how the intervention packages are implemented and the extent to which they become embedded in routine systems and practice

- Explore whether the interventions worked as predicted or by an alternative means and

- Identify unintended consequences of the delivery and implementation of the intervention packages in practice.

\section{Recruitment and consent}

We will recruit a subset of general practices not involved in the trials. Email invitations will be followed up by telephone calls and practice-level informed consent obtained. Practices will be reimbursed for their time and commitment over the study period.

We aim to recruit eight practices, providing a sample of two practices per clinical topic. We will purposively select practices to ensure a spread across CCGs and a range of practice sizes (patient list size and number of practice staff). Where individual practice staff members participate in audio-recorded interviews, individual written consent will be obtained.

\section{Method}

Process evaluation practices will be randomised by the trial statistician to receive an intervention package targeting one of the four indicators. These will be delivered in identical fashion to, and in parallel with, the trial practices.

In preparation for data collection at the process evaluation practices, we will develop a logic model to capture how we expect the intervention package and its constituent components to be received and how it may bring about change [22, 23].

Throughout the intervention period, we will conduct indepth work with process evaluation practices to observe and monitor how the interventions are received and implemented. Data collection will proceed via observations, interviews and the collection of administrative data [24].

- Observations: where possible, settings where practice staff discuss or interact with the intervention package and/or clinical topic will be observed (e.g. educational outreach sessions, practice meetings), and detailed field notes generated to capture how staff receive and interact with the intervention package at a group level [25]. No individual patient consultations or data will be collected.

- Interviews: staff will be interviewed individually about their role in the practice, their interaction with the intervention, whether it is perceived to influence behaviour in relation to the clinical topic, if and how the intervention has been incorporated into routine practice, and any unintended consequences arising from the intervention. Interviews will be both semi-structured and open-ended to help capture intended and unintended mechanisms. A range of practice staff will be interviewed in each participating practice (up to 12 individuals per site), including staff with administrative, clinical and managerial roles. Interviews will take place at three time points, twice to coincide with delivery of key intervention components (educational outreach and feedback report delivery) and, finally, after trial end. Interviews will be audio-recorded and transcribed verbatim.

- Administrative data: information relating to the intervention package and/or target clinical recommendations will be collected. Examples include meeting minutes, practice protocols around a clinical topic and communications (e.g. emails) about the clinical topic or intervention package.

\section{Analysis}

Data will be collated to produce chronological reconstructions of the process and outcomes of receiving and implementing the intervention package. This will be on a case by case basis, wherein each practice acts as a case study [26]. Data will be analysed using a framework approach [27] based on our pre-specified theories of how the intervention package will work (outlined in the logic model) and drawing on normalisation process theory to understand the process of implementation in each practice [28]. We also aim to identify and explore any unintended consequences and mechanisms of the intervention package during analysis.

\section{Ethical review}

Ethical approval has been obtained through the UK National Research Ethics Service (14/SC/1393) (Additional file 2). NHS Research and Development approvals were granted by the NHS Yorkshire \& Humber Commissioning Support Unit on behalf of all the CCGs in West Yorkshire (approved 30 January 2015).

\section{Trial status}

The trials are currently in progress. No endpoint data collection nor cleaning has yet occurred. 


\section{Discussion}

The ASPIRE trials will provide robust evidence about the effectiveness and cost-effectiveness of an implementation package for primary care targeting 'high impact' indicators, as well as the further insights generated by the process evaluation.

\section{Additional files}

Additional file 1: CONSORT 2010 checklist of information to include when reporting a cluster randomised trial. (DOCX 28 kb)

Additional file 2: Confirmation of ethical approval. (PDF $262 \mathrm{~kb}$ )

\section{Abbreviations}

ACE-l: angiotensin-converting-enzyme inhibitor; ACR: albumin to creatinine ratio; ARB: angiotensin receptor blocker; ASPIRE: Action to Support Practices Implementing Research Evidence; CCG: clinical commissioning group; CHD: coronary heart disease; CKD: chronic kidney disease; CONSORT: Consolidated Standards of Reporting Trials; COPD: chronic obstructive pulmonary disease; CRN: Clinical Research Network; eGFR: estimated glomerular filtration rate; GP: general practitioner; ICC: intra-cluster correlation coefficient; IFCC-HbA1c: International Federation of Clinical Chemistry and Laboratory Medicine-Glycated Haemoglobin; IMD: index of multiple deprivation; ITT: intention-to-treat; LICTR: Leeds Institute of Clinical Trials Research; NHS: National Health Service; NICE: National Institute for Health and Care Excellence; NIHR: National Institute for Health Research; NRES: National Research Ethics Service; NSAID: non-steroidal anti-inflammatory drug; PAD: peripheral arterial disease; PCR: protein to creatinine ratio; QALYs: qualityadjusted life years; QOF: Quality and Outcomes Framework; REC: Research Ethics Committee; TIA: transient ischemic attack; TMG: Trial Management Group; TSC: Trial Steering Committee; UK: United Kingdom.

\section{Competing interests}

RF and LG are editors of Implementation Science. All decisions about this manuscript were made by another editor. The authors declare that they have no other competing interests.

\section{Authors' contributions}

TW, SH, LG, AJF, RL, RMc, PH, SC and RF designed the study. CH, DM and DB are responsible for the health economics assessment. TW, SH. LG, El, PH and MC are responsible for the operational delivery of the trials. $\mathrm{PH}, \mathrm{MC}$ and $\mathrm{PC}$ are primarily responsible for data acquisition. $\mathrm{PH}, \mathrm{MC}$ and $\mathrm{AJF}$ are responsible for statistical analysis and the reporting of the trials. LG, CH and WW designed and are responsible for the process evaluation. RF is the Principal Investigator for the trials and was involved in every step of study conception, design and manuscript editing. All authors read and approved the final manuscript.

\section{Acknowledgements}

The ASPIRE programme team comprises Robert M West, Martin Rathfelder, Judith Richardson, Tim Stokes and lan Watt, in addition to the named authors. We also acknowledge the contributions of Andrew Davies, Chris Jackson, John Turgoose, Duncan Petty, Stella Johnson and Sarah Alderson.

\section{Funding and trial administration}

This paper presents independent research funded by the National Institute for Health Research (NIHR) under its Programme Grants for Applied Research scheme (grant Reference Number RP-PG-1209-10040). The views expressed in this paper are those of the authors and not necessarily those of the NHS, the NIHR or the Department of Health. The trials are sponsored by the University of Leeds and are co-ordinated by the Leeds Institute of Health Sciences and the Leeds Institute of Clinical Trials Research. The trial management group consists of the co-applicants and teams from the co-ordinating Institutes. The trial is adopted by the UK Clinical Research Network (CRN). The trial is registered (ISRCTN91989345).

\section{Author details}

${ }^{1}$ Leeds Institute of Health Sciences, University of Leeds, Leeds LS2 9JT, UK.

${ }^{2}$ Leeds Institute of Clinical Trials Research, University of Leeds, Leeds LS2 9JT,
UK. ${ }^{3}$ School of Psychology, University of Leeds, Leeds LS2 9LZ, UK. ${ }^{4}$ Bradford Institute for Health Research, Bradford Royal Infirmary, Bradford BD9 6RJ, UK. ${ }^{5}$ West Yorkshire Research Service, Bradford Districts Clinical Commissioning Group, Douglas Mill, Bradford BD5 7JR, UK.

Received: 10 February 2016 Accepted: 20 February 2016

Published online: 29 February 2016

\section{References}

1. Cooksey R. A review of UK health research funding. London: HMSO; 2006.

2. Dawda P, Jenkins R, Varnam R. Quality improvement in general practice. London: The King's Fund; 2010.

3. Roland M. Linking physicians' pay to the quality of care-a major experiment in the United Kingdom. N Engl J Med. 2004;351(14):1448-54. doi:10.1056/NEJMhpr041294.

4. Rushforth B, Stokes T, Andrews E, Willis TA, McEachan R, Faulkner S, et al. Developing 'high impact' guideline-based quality indicators for UK primary care: a multi-stage consensus process. BMC Fam Pract. 2015;16(1):156.

5. Campbell SM, Reeves D, Kontopantelis E, Sibbald B, Roland M. Effects of pay for performance on the quality of primary care in England. N Engl J Med. 2009;361(4):368-78. doi:10.1056/NEJMsa0807651.

6. Eccles M, Grimshaw J, Campbell M, Ramsay C. Research designs for studies evaluating the effectiveness of change and improvement strategies. Qual Saf Health Care. 2003;12(1):47-52. doi:10.1136/qhc.12.1.47.

7. Grimshaw JM, Thomas RE, MacLennan G, Fraser C, Ramsay CR, Vale $L$ et al. Effectiveness and efficiency of guideline dissemination and implementation strategies. Health Technol Assess. 2004;8(6):iii-iv, 1-72.

8. Office for National Statistics. Population estimates for UK, England and Wales, Scotland and Northern Ireland, mid-2014. 2015. http://www.ons.gov. uk/ons/publications/re-reference-tables.html?edition=tcm\%3A77-368259. Accessed 26 January 2016.

9. Loudon K, Treweek S, Sullivan F, Donnan P, Thorpe KE, Zwarenstein M. The PRECIS-2 tool: designing trials that are fit for purpose. BMJ. 2015;350. doi:10. 1136/bmj.h2147.

10. Jamtvedt G, Young JM, Kristoffersen DT, O'Brien MA, Oxman AD. Audit and feedback: effects on professional practice and health care outcomes. Cochrane Database Syst Rev. 2006;2, CD000259.

11. O'Brien MA, Rogers S, Jamtvedt G, Oxman AD, Odgaard-Jensen J, Kristoffersen DT, et al. Educational outreach visits: effects on professional practice and health care outcomes. Cochrane Database Syst Rev. 2007:4, CD000409.

12. Shojania KG, Jennings A, Mayhew A, Ramsay CR, Eccles MP, Grimshaw J. The effects of on-screen, point of care computer reminders on processes and outcomes of care. Cochrane Database Syst Rev. 2009; (3):Cd001096. doi:10. 1002/14651858.CD001096.pub2.

13. National Collaborating Centre for Chronic Conditions. Type 2 diabetes: national clinical guideline for management in primary and secondary care (update). London: Royal College of Physicians; 2008.

14. National Institute for Health and Care Excellence. Type 2 diabetes in adults: management. London: Royal College of Physicians; 2015.

15. Dreischulte T, Grant A, Donnan P, McCowan C, Davey P, Petrie D, et al. A cluster randomised stepped wedge trial to evaluate the effectiveness of a multifaceted information technology-based intervention in reducing highrisk prescribing of non-steroidal anti-inflammatory drugs and antiplatelets in primary medical care: The DQIP study protocol. Implement Sci. 2012;7:24.

16. National Clinical Guideline Centre. Hypertension: the clinical management of primary hypertension in adults. London: Royal College of Physicians; 2011.

17. National Institute for Health and Care Excellence. Atrial fibrillation: the management of atrial fibrillation [CG180]. London: National Institute for Health and Care Excellence; 2014

18. Michie S, Johnston M, Abraham C, Lawton R, Parker D, Walker A. Making psychological theory useful for implementing evidence based practice: a consensus approach. Qual Saf Health Care. 2005;14(1):26-33. doi:10.1136/ qshc.2004.011155.

19. Doran T, Fullwood C, Gravelle H, Reeves D, Kontopantelis E, Hiroeh U, et al. Pay-for-performance programs in family practices in the United Kingdom. N Engl J Med. 2006;355(4):375-84. doi:10.1056/NEJMsa055505.

20. Grilli R, Lomas J. Evaluating the message: the relationship between compliance rate and the subject of a practice guideline. Med Care. 1994;32(3):202-13.

21. Rogers E. Diffusion of innovations. New York: Free Press; 1995. 
22. Cooksy L, Gill P, Kelly PA. The program logic model as an integrative framework for a multimethod evaluation. Eval Program Plann. 2001;24(2): 119-28. doi: 10.1016/50149-7189(01)00003-9.

23. Patton M. Utilization-focused evaluation. Fourth edition. Thousand Oaks: Sage; 2008.

24. Mays N, Pope C. Qualitative research: observational methods in health care settings. BMJ. 1995;311(6998):182-4.

25. Emerson R, Fretz R, Shaw L. Writing ethnographic fieldnotes. 2nd ed. Chicago: The University of Chicago Press; 2011.

26. Luck L, Jackson D, Usher K. Case study: a bridge across the paradigms. Nurs Inq. 2006;13(2):103-9. doi:10.1111/j.1440-1800.2006.00309.x.

27. Gale NK, Heath G, Cameron E, Rashid S, Redwood S. Using the framework method for the analysis of qualitative data in multi-disciplinary health research. BMC Med Res Methodol. 2013;13:117. doi:10.1186/1471-2288-13-117.

28. May C, Finch T. Implementing, embedding, and integrating practices: an outline of normalization process theory. Sociology. 2009;43(3):535-54. doi: $10.1177 / 0038038509103208$

Submit your next manuscript to BioMed Central and we will help you at every step:

- We accept pre-submission inquiries

- Our selector tool helps you to find the most relevant journal

- We provide round the clock customer support

- Convenient online submission

- Thorough peer review

- Inclusion in PubMed and all major indexing services

- Maximum visibility for your research

Submit your manuscript at www.biomedcentral.com/submit 